\title{
EVALUACIÓN DE LA SACHARINA SECA (CAÑA ENRIQUECIDA) COMO SUPLEMENTO EN LA ALIMENTACIÓN DE VACAS LECHERAS EN LA ÉPOCA SECA ${ }^{1}$
}

\author{
Reina Zamora ${ }^{2}$, Romeo Solano ${ }^{3}$
}

\begin{abstract}
RESUMEN
Evaluación de la Sacharina Seca (Caña enriquecida) como suplemento en la alimentación de vacas lecheras en la época seca. El presente trabajo fue realizado en la Finca "El Hato Ganadero", ubicada en la ciudad de Estelí, Región I de Nicaragua. Se evaluó la utilización de la sacharina (caña enriquecida) como suplemento en la alimentación de vacas lecheras durante un período experimental de 64 días de la época seca (enero a marzo de 1992). Se usó un diseño estadístico de sobrecambio con cuatro subperíodos de 14 días cada uno. Se utilizaron seis vacas (tres por grupo), de genotipo cruzado (Pardo Suizo x Cebú), seleccionadas según su estado de lactancia, edad, producción de leche y fecha de parto. Las vacas del grupo experimental, recibieron como suplemento alimenticio $4,12 \mathrm{~kg}$ de sacharina, ofrecidos diariamente a libre acceso, después del ordeño. El grupo control recibió la alimentación ofrecida por el productor, al resto del hato en producción. Al inicio de la prueba, las vacas presentaron un promedio de $500 \mathrm{~kg}$ de peso vivo y una producción de 8 litros de leche, en un ordeño al día. Los resultados obtenidos indican que el efecto de la sacharina en la producción de leche fue altamente significativa $(\mathrm{P}<0,01)$, con respecto al tratamiento control, por lo que se deduce que su utilizacion como suplemento alimenticio para este tipo de ganado es recomendable. El análisis económico presentó un beneficio neto marginal de C $\$ 3.36$ y una tasa de retorno marginal del $339 \%$, por lo que el uso de la caña de azúcar (Saccharum officinarum) en forma de sacharina, es una buena alternativa como suplemento alimenticio para el ganado lechero de la Cuenca de Estelí.
\end{abstract}

\begin{abstract}
Evaluation of dry saccharine as feed supplement for dairy cows during the dry season. This research was conducted in Region I in Nicaragua to evaluate the use of saccharine (enriched sugar can e) as feed supplement for dairy cattle on a 65 day experimental period during the dry season, using the statistical design of overchange, with four sub-periods of 14 days each. Six (three per group) crossed-bred cows (Brown Swiss x Zebu) were used. They were selected according to their lactating period, age, milk produc-tion and calving date. Before starting the trial, the cows weighed an average of $500 \mathrm{~kg}$ and were producing 8 liters of milk per day, in one milking. The experimental group was fed (ad libitum) $4.12 \mathrm{~kg}$ of saccharine, after the milking. The results show that the effect of the saccharine on the milk production was highly significant $(\mathrm{P}>0.01)$ therefore we con-cluded that the use of saccharine as a feed supplement for this type of cattle is convenient and recommendable.
\end{abstract}

\section{INTRODUCCION}

En la región I de Nicaragua, como en todo el trópico seco de Centro América, durante la época de verano (noviembre-mayo) se presenta uno de los principales problemas para la producción ganadera debido a la fuerte escasez de alimentos para el ganado, en por lo menos 4 meses del año.

\footnotetext{
1 Presentado en la XXXIX Reunión Anual del PCCMCA en Guatemala, América Central. 28 de marzo - 3 de abril, 1993.

2 Zootecnista, Responsable del Dpto. de Pastos y Alimentación, Comisión Regional de Ganadería I. (Nicaragua)

3 Agrónomo Zootecnista, Líder de los Proyectos Agroforestales CATIE/SAREC. (Turrialba, Costa Rica)
} 
Una buena opción para ser utilizada como alimento en el verano es la caña de azúcar (Sacharum officinarum), la cual es una planta bien adaptada a gran variedad de suelos en la región, rica en carbohidratos (68\% BS), con una alta digestibilidad de la materia orgánica $(78,78 \%)$, lo que unido al bajo contenido de proteína bruta $(0.80)$ hace factible la inclusión de la uréa u otra fuente de nitrógeno no protéico (NNP) para lograr síntesis de proteína microbiana y mejorar la respuesta animal (Alvarez, 1986 y Elías et al., 1990).

En Cuba y México se ha desarrollado una tecnología práctica que consiste en el enriquecimiento protéico de lacañade azúcar, mediante un proceso que transforma paIte de los carbohidratos disponibles en proteínas verdaderas con la intervención y reproducción de levaduras que existen naturalmente en la caña.

La necesidad de buscar soluciones parciales a la escasez de alimentos para el ganado bovino de las zonas supeditadas a fuertes sequías anuales, a través de opciones prácticas y adaptadas a las condiciones socioeconómicas de los ganaderos de pequeñas y medianas explotaciones y con el fin de propiciar el uso de recursos factibles de ser producidos en la finca, originó la conducción del presente trabajo, con los siguientes objetivos:

- Determinar la factibilidad técnica y económica de la elaboración y uso de sacharina seca como suplemento en la alimentación de vacas lecheras,. en condiciones de finca del productor.

- Conocer la composición química proximal y digestibilidad in vitro de la sacharina seca.

- Evaluar el efecto de la sacharina seca en la producción de leche de vacas cruzadas (Parado Suizo x Cebú).

\section{MATERIALES Y MÉTODOS}

\section{Ubicación}

El ensayo se llevó a cabo en la finca "El Hato Ganadero" ubicado en Paso de León en la ciudad de
Estelí, Nicaragua, a $850 \mathrm{msnm}$, con temperatura promedio anual de $23,8^{\circ} \mathrm{C}$ y $900 \mathrm{~mm}$ de precipitación anual, distribuida irregularmente durante seis meses del año. En Estelí, la deficiencia de agua para los cultivos se presenta desde finales del mes de noviembre hasta el mes de abril inclusive, ocasionando una marcada escasez de alimentos forrajeros para el ganado bov del mes de noviembre hasta el mes de abril inclusive, ocasionando una marcada escasez de alimentos forrajeros para el ganado bovino (Figura 1).

\section{Diseño experimental}

Se utilizó un diseño estadístico de sobrecambio con dos grupos de tres vacas cada uno y cuatro periodos experimentales de 14 días, según el modelo estadístico siguiente:

$$
\text { YIJKL }=\mathrm{U}+\mathrm{Ti}+\mathrm{Vj}+\mathrm{Pk}+\text { Eijkl, donde: }
$$

$\mathrm{Y}=$ producción de leche de la vaca sometida al;

$\mathrm{Ti}=$ iésima dieta $(1,2)$;

$\mathrm{Vj}=$ Jésima vaca $(1,6)$;

$\mathrm{Pk}=$ Késimo periodo $(1,4)$;

Eijkl = errores aleatorios, distribuidos normalmente con media 0 y varianza Donde cada vaca fue considerada como una unidad experimental bajo un manejo de sobrecambio.

Los tratamientos consistieron en:

$\mathrm{TA}=$ Tratamiento Control, consistente en vacas alimentadas con el suplemento del productor, quien ofreció diariamente: $3,14 \mathrm{~kg}$ de maíz y 0,26 $\mathrm{kg}$ de frijol molidos (ambos de desecho), des pues del ordeño.

$\mathrm{TB}=$ Vacas alimentadas con el Tratamiento A (control) más 4,12 kg de sacharina seca, ofrecida una vez al día, despues del ordeño.

Los períodos experimentales fueron de 14 días: seis para adaptación y ocho para la toma de datos. El experimento se extendió 64 días de la época seca (enero y marzo). 


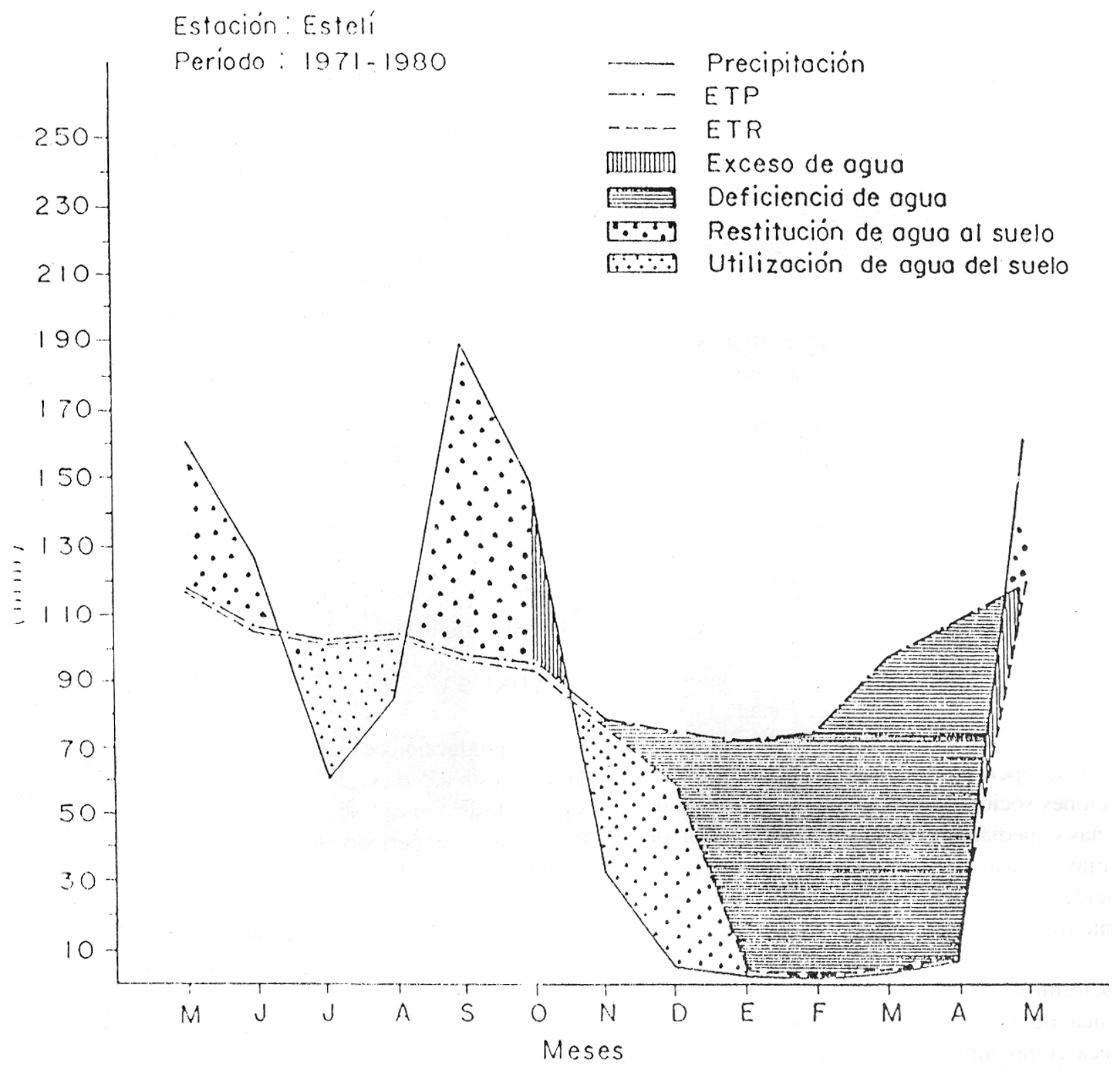

Figura 1. Balance hídrico de la Región de Estelí, segú 'Thornthwaite.

\section{Animales}

Se utilizaron seis vacas cruzadas (Pardo Suizo $\mathrm{x}$ Cebú) seleccionadas según eslado de lactancia, producción de leche, edad y fecha de parto. Los animales se encontraban entre la segunda y tercera lactación, tenían entre tres y seis meses de paridas cuando se inició el período experimental.

\section{Características de los alimentos}

El tratamiento control consistió en ma íz más frijol molidos en molino de martillos. A las vacas se les suministraron $3,40 \mathrm{~kg}$ de la mezcla $(3,14 \mathrm{y} 0,26 \mathrm{~kg}$ respectivamente) por animal una vez al día, después del ordeño. El ordeño se realizó en forma manual una vez al día, entre las 5 y 7 horas de la mañana. 
La sacharina fue elaboraba en la finca del productor, con su participación.

\section{Manejo}

Las vacas del grupo A (testigo), consumieron $3,40 \mathrm{~kg}$ diarios de suplemento utilizado por el productor en la finca, compuesto de una mezcla molida de maiz y frijol, en una proporción de 3,14 y 0,26 kg, respectivamente.

Las vacas del tratamiento $B$, se sometieron a un consumo restringido de sacharina seca. Este suplemento se ofreció balanceando raciones para cinco vacas con 500 $\mathrm{kg}$ de peso vivo, para una producción de leche diaria estimada de $10 \mathrm{~kg}$ con 3,5\% de grasa. Los cálculos se hicieron siguiendo las recomendaciones del NRC (1984). A estos requerimientos se les adicionó un $10 \%$ por el ejercicio ocasionado por el pastoreo. Se.les suministraron $4,12 \mathrm{~kg}$ de sacharina una vez por día, después del ordeño.

A una vaca de $600 \mathrm{~kg}$ para una producción de leche de $11 \mathrm{~kg}$ de leche por día, con 3,5\% de grasa se le suministraron $5,7 \mathrm{~kg}$ de sacharina una vez por día, después del ordeño. La cantidad de sacharina ofrecida equivale al $40 \%$ del consumo de materia seca diario de las vacas.

Una vez terminada la suplementación alimenticia de los dos grupos experimentales, en los comederos, las vacas se trasladaban a un potrero con población predominantemente de pasto estrella (Cynodon nlemfuensis) y un mínimo de guinea (Panicum maximum) donde pastoreaban libremente junto al resto del hato en producción de la finca, por el resto del día.

\section{Medidas}

Los rendimientos de leche fueron registrados una vez que los animales pasaron su período de adaptación realizándose 3 veces (primero, cuarto y octavo día) durante los ocho días del período experimental, en cada subperíodo.
De los alimentos ofrecidos se tomaron muestras para determinación de composición química proximal y la digestibilidad in vitro de la Materia Seca (según metodología prupuesta por Goering y Van Soest, 1970 y Kass y Rodríguez, 1990).

La EM de las raciones se calculó a partir de la materia digestible y $\%$ de ceniza de los alimentos (según metodología de cálculo propuesta por la FAO, 1989).

Mediante la metodología de presupuestos parciales descrita por el CIMMYT (1988), se realizó la evaluación económica de los tratamientos, determinando el beneficio neto marginal y la tasa de retorno marginal del ensayo bajo las condicones en que se desarrolló el trabajo, que corresponden a las imperantes en la finca del productor y a la cuenca lechera de Estelí.

\section{RESULTADOS Y DISCUSION}

La sacharina ofrecida a los animales, fue totalmente consumida, lo que coincide con los resultados encontrados por Ruíz et al (1990), quienes demostraron que la sacharina puede ser utilizada sustituyendo niveles del $40 \%$ de la materia seca (MS) de la ración, sin afectar el consumo de la misma por el animal.

La digestibilidad de la materia orgánica de la sacharina fue del 60,27\%, siendo similar a la encontrada en la caña de azúcar sin ningún tratamiento $(61,25) \mathrm{Y}$ mayor a la del maíz y pasto estrella utilizados en la alimentación del ganado (Cuadro 1). Puede observarse que la sacharina alcanza valores de proteína bruta de $14,69 \%$ y la energía bruta exhibe valores de 3,87 MCal/kg de MS, nivel protéico muy superior a los de la caña sin ningún tratamiento. La fibra bruta encontrada fué del 26,50\% lo que coincide con Elías et al. (1990), quienes destacan que la mayor parte de la fibra bruta esta formada por celulosa y hemicelulosa. En este sentido, la inclusión de sacharina en la ración mantiene valores altos de consumo y es importante resaltar el incremento en el contenido de proteína cruda que se logra cuando la caña se convierte en sacharina $(0.80 \%$ a $14.89 \%)$ (Cuadro 1$)$. 
Cuadro 1. Digestibilidad in vitro, porcentaje de proteína cruda, pared celular y materia orgánica de los alimentos utilizados en el experimento.

\begin{tabular}{lcccc}
\hline Muestras & $\begin{array}{c}\text { \% Proteína } \\
\text { cruda }\end{array}$ & \% Digest. & $\begin{array}{c}\text { \% Pared } \\
\text { celular }\end{array}$ & $\begin{array}{c}\text { \% Materia } \\
\text { orgánica }\end{array}$ \\
\hline Caña & 0,80 & 61,2 & 51,22 & 78,78 \\
Maíz & 5,92 & 51,0 & 72,07 & 85,30 \\
Pasto estrella & 7,80 & 37,7 & 73,81 & 87,93 \\
Sacharina seca & 14,69 & 60,27 & 55,49 & 95,06 \\
\hline
\end{tabular}

La digestibilidad in vitro obtenida en la sacharina fue un poco menor a la informada por Ruíz et al. (1990), quienes encontraron un rango del 62 al $68 \%$ de digestibilidad aparente en raciones para carneros con el $46 \%$ de sacharina. Probablemente esta diferencia se deba a la técnica utilizada para la determinación de la digestibilidad. Se puede observar que la digestibilidad y la proteína cruda es mayor en la sacharina que en el maíz y el pasto utilizado para alimentar a los animales durante el período de experimentación.

La digestibilidad de los nutrimentos y en particular la de la materia orgánica, permitió calcular concentraciones energéticas en las raciones del TA (maíz + frijol molidos) y TB (TA + sacharina), de alrededor de 2,9 y 3,25 MCal de $\mathrm{EM} / \mathrm{kg} \mathrm{MS}$, respectivamente.

En el Cuadro 2 se presenta la composición química de la sacharina, según análisis bromatológico realizado.

Se puede observar (en los Cuadros 1 y 2), que la sacharina presentó valores de proteína bruta mayores que el maíz y pasto estrella y es comparable con concentrados comerciales utilizados para vacas lecheras (16\% PB). La energía bruta exhibió valores de $3.87 \mathrm{MCal} / \mathrm{kg} \mathrm{MS}$. El nivel de fibra bruta fue de $26,50 \%$, destacándose que la mayor parte está formada por celulosa y hemicelulosa (ICA 1987).

\section{Producción de leche}

De acuerdo con el análisis de varianza (Cuadro 3), los tratamientos y las vacas presentaron diferencias estadísticas altamente significativas $(\mathrm{P}<0,01)$, mientras que los períodos fueron significativamente diferentes entre sí $(\mathrm{P}<0,05)$.

Del análisis de varianza se deduce que el diseño utilizado fue eficiente en separar de la varianza experimental la correspondiente a las causas de variación anotadas.

El efecto de la sacharina fue altamente diferente ( $\mathrm{P}$ $<0,01)$, por lo que se deduce que su utilización como suplemento alimenticio para este tipo de ganado es conveniente y recomendable, pues las diferencias entre medias fue de 1,743 litros por vaca por día ( $\mathrm{A}=8,443$; $\mathrm{B}$ $=10,186)$.

La diferencia anterior se esperaba mayor según la ración calculada, pero indudablemente la condición del pastizal al que tenía acceso el ganado, fue disminuyendo en cantidad y calidad al avanzar la época de sequía y con ella el experimento, evitando una recolección más completa de alimentc proveniente del pasto, por parte de las vacas en pastoreo.

La Figura 2 presenta la diferencia en producción de leche de los dos grupos (con y sin sacharina) de vacas sometidas al ensayo. Puede observarse el ascenso y caída del grupo de vacas que no recibió sacharina (TA). 
Cuadro 2. Composición bromatológica de la sacharina.

\begin{tabular}{lr}
\hline Sacharina & $\%$ \\
\hline Materia seca & 87,73 \\
Proteína bruta & 14,69 \\
Ceniza & 4,38 \\
Fibra bruta & 26,50 \\
Calcio & 0,35 \\
Fósforo & 0,25 \\
Potasio & 0,05 \\
Magnesio & 0,20 \\
EB (MCal/kg MS) & 3,87 \\
Extracto Etereo & 1,10 \\
\hline
\end{tabular}

Mientras que el grupo que fue alimentado con sacharina como suplemento exhibió un comportamiento lineal y ascendente durante los cuatro períodos experimentales, resaltándose el beneficio de la sacharina en la producción ascendente de leche.

\section{Evaluación económica}

Se calculó el valor por kilogramo de sacharina seca, partiendo de la base del costo de la tonelada métrica de caña de azúcar en la región, el costo de la urea, sales minerales y mano de obra utilizada para su elaboración. El costo por kilogramo de sacharina producida en la finca fue de $\mathrm{C} \$ 0,24$ (Cuadro 4).
De acuerdo al análisis bromatológico de la sacharina (Cuadro 2), de una tonelada de sacharina fresca se obtienen $877,30 \mathrm{~kg}$ de sacharina seca, por lo tanto, el costo por kilogramo de sacharina seca fue de $\mathrm{C} \$ 0,24$.

En el Cuadro 5 se puede observar que hubo un aumento en el promedio de producción de leche $(1,741)$ cuando las vacas fueron suplementadas con sacharina, exhibiendo un incremento neto de C $\$ 4,35 /$ vaca/día. Puede observarse también, la ventaja de la respuesta de la sacharina, pues no solamente convierte el proceso de producción más económico sino que presenta una alternativa de alimentación de vacas lecheras que puede prescindir de la utilización de alimentos destinados para la nutrición del agricultor y su familia, los cuales pueden alcanzar en el mercado, precios de oportunidad mayores.

La tasa de retorno marginal, encontrada de 339,39\% es plenamente representativa de la con veniencia de utilizar esta alternativa de alimentación de ganado lechero en época seca, en este tipo de ganado, bajo las circunstancias sociales y económicas de los productores de leche de la cuenca lechera del valle de Estelí.

\section{CONCLUSIONES}

En base a los resultados obtenidos se puede concluir que la sacharina es técnica y económicamente factible para ser utilizada como suplemento en alimentación de

Cuadro 3. Análisis de varianza.

\begin{tabular}{lcccc}
\hline F de V & G.L. & SC & CM & F \\
\hline Vacas & 5 & 37,3777 & 7,4755 & $14,46^{* *}$ \\
Períodos & 3 & 6,1539 & 2,0513 & $3,96^{*}$ \\
Tratamientos & 1 & 18,2302 & 18,2302 & $35,27^{* *}$ \\
Error & 14 & 7,2360 & 0,5169 & \\
TOTAL & 23 & 68,9978 & & \\
\hline
\end{tabular}

* Significativamente diferentes $(\mathrm{P}<0,05)$

** Altamente significativas $(\mathrm{P}<0,01)$ 


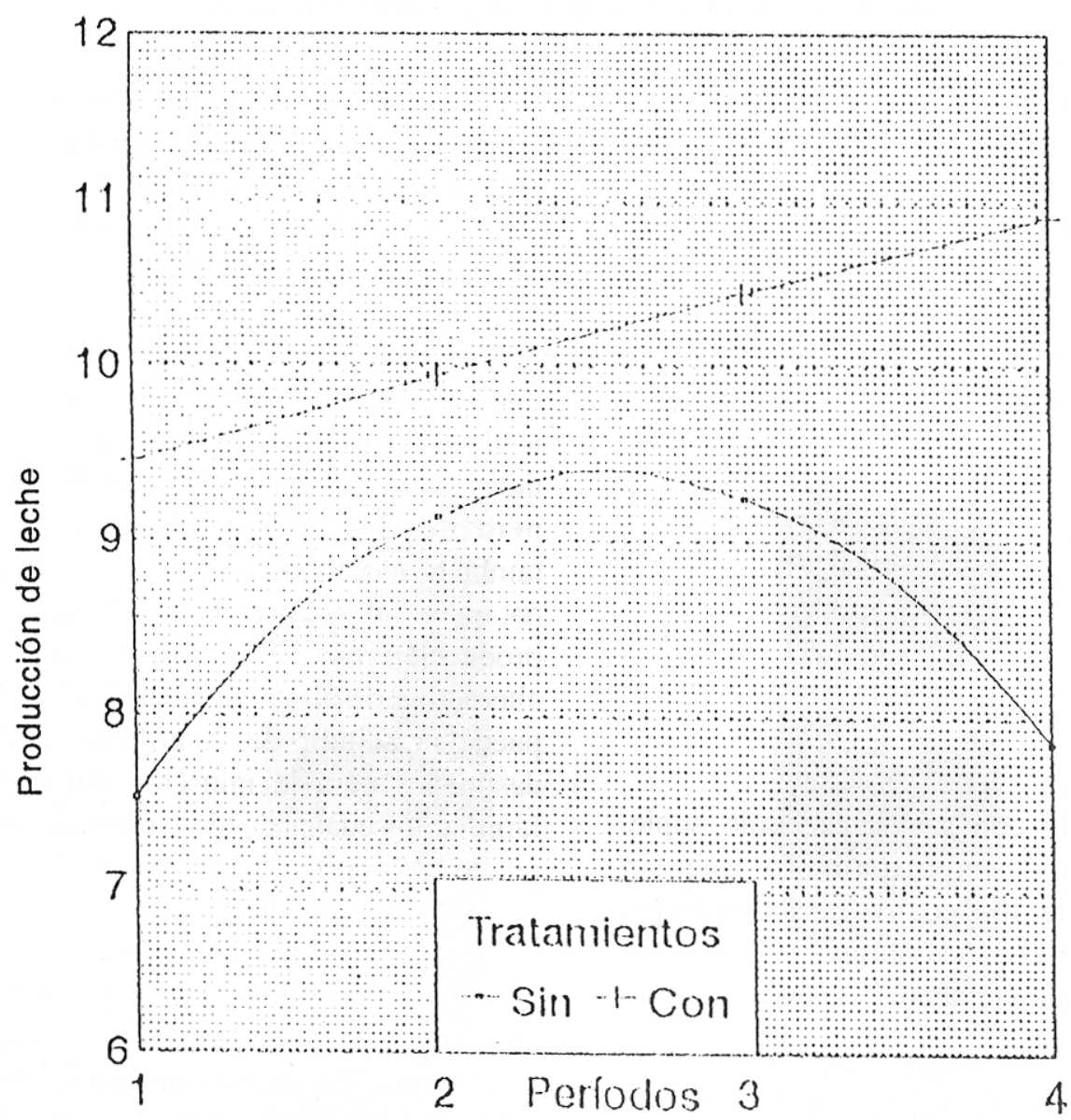

Figura 2. Producción de leche, $\mathrm{kg} / \mathrm{vac} a / \mathrm{d}$ á, por periodo para labamicntes con y sin sacharina.

Cuadro 4. Costo de preducción de sacharina seca (C $\$ / 1)$ (Cóndovas (Oo 5x 1 Dular Anericano).

\begin{tabular}{|c|c|c|c|c|}
\hline$\Lambda$ C'IIVID $\Lambda$ IDES & $\mathrm{U} / \mathrm{M}$ & Canlidad & $\begin{array}{c}\text { Costo } \\
\text { Unitario }\end{array}$ & $\begin{array}{l}\text { Costo } \\
\text { 'rotal }\end{array}$ \\
\hline \multicolumn{5}{|l|}{ Matcria prima } \\
\hline Caña de azúcar & 'TM & 1 & $120,(0)$ & $120,(0)$ \\
\hline Corte & h/hombre & 1 & $2,(0)$ & $2,(0)$ \\
\hline Urea & $\mathrm{kg}$ & 15 & 1,58 & 23,70 \\
\hline Sales mincrales & $\mathrm{kg}$ & 5 & 4,48 & 49,26 \\
\hline Plástico & $111^{2}$ & 11 & $12,(1) 0$ & $12,00)$ \\
\hline \multicolumn{5}{|l|}{ Mano de obra } \\
\hline Picado, Eliboración & h/hombre & 4 & $2,(1)$ & $8,(0)$ \\
\hline Voltco & h/hombre. & 8 & $2,(0)$ & $16,0)$ \\
\hline Desintegrado & h/hombre & 2 & $2,()()$ & 4,00 \\
\hline Carreta de bucy & horal & 1 & $5,(0)$ & $5,(0)$ \\
\hline TO'TAL & & & & C.\$24(1), (0) \\
\hline
\end{tabular}

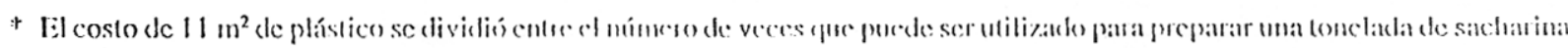
a la vez. 
Cuadro 5. Análisis económico de la suplementación con sacharina.

\begin{tabular}{ccc}
\hline Concepto & Tratamiento & Tratamiento \\
& A & B \\
\hline
\end{tabular}

\begin{tabular}{|c|c|c|}
\hline \\
\hline & & \\
\hline $\begin{array}{l}\text { Promedio total de } \\
\text { producción/lt leche }\end{array}$ & 8,44 & 10,18 \\
\hline Precio de leche $C \$$ & 2,50 & 2,50 \\
\hline Ingreso por venta $C \$$ & 21,10 & 25,45 \\
\hline Total Ingresos & 21,10 & 25,45 \\
\hline \multicolumn{3}{|c|}{ (B) Costos Incrementales } \\
\hline \multicolumn{3}{|c|}{ Maíz molido: $3,14 \mathrm{~kg} \mathrm{x}$} \\
\hline $\mathrm{C} \$ 0,66$ & 2,072 & 2,072 \\
\hline Frijol: $0,27 \mathrm{~kg} \mathrm{x}$ & & \\
\hline $\mathrm{C} \$ 0,77$ & 0,208 & 0,208 \\
\hline \multicolumn{3}{|l|}{ Sacharina: $4,12 \mathrm{~kg} \mathrm{x}$} \\
\hline $\mathrm{C} \$ 0,24$ & - & 0,990 \\
\hline Total & 2,280 & 3,270 \\
\hline \multicolumn{3}{|c|}{ (C) Producción Incrementada } \\
\hline Lts/vaca/día & 1,74 & \\
\hline \multicolumn{2}{|c|}{ (D) Beneficio Incremental/Vaca/día } & 4,35 \\
\hline (E) Beneficio Neto & 18,82 & 22,18 \\
\hline \multicolumn{2}{|c|}{ (F) Beneficio Neto Marginal } & 3,36 \\
\hline \multicolumn{2}{|c|}{ (G) Tasa de Retorno Marginal } & $339,39 \%$ \\
\hline
\end{tabular}

vacas lecheras durante la época de verano. Además de mantener, incrementa la producción de leche en los animales, haciendo de esta manera rentable la in versión.

\section{RECOMENDACIONES}

a. Se recomienda la utilización de la sacharina como suplemento alimenticio en raciones de ganado lechero con características similares al existente en el hato donde se realizó el presente estudio. b. Se recomienda este estudio en oro tipo de ganado como el predominante de la zona con alto encaste cebuíno y en condiciones de manejo y alimentación comunes en la región.

\section{LITERATURA CITADA}

CHURCH, D. C. 1974. Fisiología digestiva y nutrición de los rumiantes. Trad. por P. Ducar Maluenda, S. Climén París y J. Camón Urgel. Zaragoza, España, ACRIBA. 3 v.

CIMMYT (MEX.) PROGRAMADEMAIZ. 1988. La formulación de recomendaciones a partir de datos agronómicos. México, D. F.

1988. La formulación de recomendaciones a partir de datos agronómicos: Un manual metodológico de evaluación económica. México D.F.

ETGEN, W.M.; REA VES, P.M. 1985. Ganado lechero, alimentación y administración. Editorial Limusa. Primera edición.

ELIAS, A.; LEZCANO, O.; LEZCANO, P.; CORDERO, J.; QUINTANA, L. 1990. Reseña descriptiva sobre el desarrollo de una tecnología de enriquecimiento proteico en la caía de azúcar mediante fermentación en estado sólido (sacharina). Revista Cubana de Ciencias Agrícolas 24(1): 1-12.

FAO. (ITALIA). 1986. Consultas de expertos de la FAO sobre la utilización de caña de azúcar como pienso. Roma.

1989. Feeding dairy cows in the tropics. Roma.

GALLEJO MARIN, M.I. 1988. Manejo del problema reproductivo en ganado de leche. Médicas Veterinaris (Col.)

GARCIA, G.Q.; NECKLES, FA; LALLO, C.H.O. 1990. Dietas basadas en forraje de caña de azúcar para la producción de carne. Revista Cubana de Ciencias Agrícolas 24(1):13-27.

GOERING, H.K., VAN SOEST, P. J., 1970. Forage fiber analisis. U.S. Department of Agriculture. Agriculture Handbook no. 379. 20 p. 
KASS, M.; RODRIGUEZ, G. 1986. Métodos de análisis rutinarios del Laboratorio de Producción Animal. Turrialba, Costa Rica, CATIE. 30 p. (mimeografiado).

LEZCANO, P.; ELIAS, A.; LAMAZARES, E.; ACHAN, J. 1990. Niveles de inclusión de sacharina en el pienso para cerdos en preceba. Revista Cubana de Ciencias Agrícolas 24(1):85-89.
RODRIGUEZ, V. 1988. Manual para la formulación y fabricación de los piensos criollos en la empresa pecuaria. La Habana, Ministerio de la Agricultura.

RUIZ, K; GAIBO,J.; MARRERO, D.; ELlAS, A. 1990. Consumo y digestibilidad en carneros alimentados con diferentes proporciones de saccharina en el concentrado. Revista Cubana de Ciencias Agrícolas 24(1):61-67. 